Soliton collision in biomembranes and nerves - A stability study.

Appali, R.; Lautrup, Benny Elley; Heimburg, Thomas Rainer; van Rienen, U.

Published in:

Journal of Mathematics in Industry

Publication date:

2012

Document version

Early version, also known as pre-print

Citation for published version (APA):

Appali, R., Lautrup, B. E., Heimburg, T. R., \& van Rienen, U. (2012). Soliton collision in biomembranes and nerves - A stability study. Journal of Mathematics in Industry, 16, 205-212. 


\title{
Soliton Collision in Biomembranes and Nerves- A Stability Study
}

\author{
Revathi Appali, Benny Lautrup, Thomas Heimburg, and Ursula van Rienen
}

\begin{abstract}
Collision of moving solitons is an interesting phenomena which is closely related to the stability of solitons. We study the head-on collision of solitons in a recently introduced model for biomembranes and nerves. We conduct simulations for pairs of solitons moving in opposite directions with the same velocity. It is found that these stable solitons collide elastically and it results a small amplitude noise traveling with higher velocity. We have also examined the energy loss of the solitons after collision.
\end{abstract}

\section{Introduction}

The functional success of electrically stimulated brain implants eg. Deep Brain Stimulation (DBS) depends on the basic understanding of signal propagation in the nerve cells. Mathematical models of pulse propagation in these cells play a major role in further investigation of the interaction of these nerve cells with the electrodes. One such mathematical description of the nerve pulse propagation is "soliton model". Soliton model is based on the propagation of a localized density wave in the axon membrane $[1,3]$. The important requirement of the model is the empirically known lipid phase transitions slightly below the physiological temperatures. Soliton models predict the exact pulse propagation velocities in myelinated nerves. The propagation velocities are closely related to the lateral sound velocities in the nerve membrane [1]. During compression, the appearance of a voltage pulse seems to be

\footnotetext{
R. Appali · U. van Rienen $(\varangle)$

Institute of General Electrical Engineering, University of Rostock, Justus-von-Liebig-Weg 2 18059 Rostock, Germany,

e-mail: revathi.appali@uni-rostock.de,ursula.van-rienen@uni-rostock.de

B. Lautrup · T. Heimburg

Niels Bohr Institute, Blegdamsvej 17, DK-2100, Copenhagen, Denmark

e-mail: lautrup@nbi.dk,theimbu@nbi.dk
} 
a consequence of the piezo-electric nature of partially charged and asymmetric cell membrane [2]. Moreover, the soliton model explains the reversible temperature and heat exchanges observed in connection with the nerve pulse. Another advantage of a soliton-based description of pulse propagation in nerves is its predictive power [1].

Lautrup et al. demonstrated that the soliton ${ }^{1}$ solutions are stable with respect to small amplitude fluctuations and robust in the presence of dissipation. This shows that the solitons can propagate under realistic physiological conditions over the length scales of nerves (upto several meters eg., sciatic nerve in human) even in the presence of friction and lateral heterogeneities [3]. In this paper, we examined the stability of the solitons with the help of collision studies, which was not considered in reference [3]. In the following section, we will discuss the model from [3].

\section{Soliton Model}

The nerve pulse propagation in a myelinated nerve can be described by (3)

$$
\frac{\partial^{2}}{\partial \tau^{2}} \Delta \rho^{A}=\frac{\partial}{\partial z}\left[\left(c_{0}^{2}+p \Delta \rho^{A}+q\left(\Delta \rho^{A}\right)^{2}+\ldots\right) \frac{\partial}{\partial z} \Delta \rho^{A}-h \frac{\partial^{4}}{\partial z^{4}} \Delta \rho^{A}\right]
$$

Here,

- $\Delta \rho^{A}$ is the change in lateral density of the membrane $\Delta \rho^{A}=\rho^{A}-\rho_{0}^{A}$.

- $\rho^{A}$ is the lateral density of the membrane.

- $\rho_{0}^{A}$ is the equilibrium lateral density.

- $c_{0}$ is the velocity of small amplitude sound.

- $\quad p$ and $q$ are the parameters determined from sound velocity and density dependence.

- $h$ is the parameter to set the linear scale of the propagating pulse.

The empirical equilibrium value of $\rho_{0}^{A}$ is $4.03510^{-3} \mathrm{~g} / \mathrm{m}^{2}$ and the low frequency sound velocity $c_{0}$ is $176.6 \mathrm{~m} / \mathrm{s}$. The coefficients $p$ and $q$ were fitted to measured values of the sound velocity as a function of density.

We work with the dimensionless variables $u, x$ and $t$ defined in [3] as

$$
u=\frac{\Delta \rho^{A}}{\rho_{0}^{A}} \quad x=\frac{c_{0}}{h} z \quad t=\frac{c_{0}^{2}}{\sqrt{h}} \tau \quad B_{1}=\frac{\rho_{0}}{c_{0}^{2}} p \quad B_{2}=\frac{\rho_{0}^{2}}{c_{0}^{2}} q
$$

Equation (1) takes the following form with these variables

\footnotetext{
${ }^{1}$ We use the term "soliton" synonymous to "solitary wave". Since the localized solutions pass through each other and dissipate some energy, which is not the case for genuine solitons.
} 


$$
\frac{\partial^{2} u}{\partial t^{2}}=\frac{\partial}{\partial x}(B(u)) \frac{\partial u}{\partial x}-\frac{\partial^{4} u}{\partial x^{4}}
$$

with

$$
B(u)=1+B_{1} u+B_{2} u^{2}
$$

Here the parameter values are chosen as $B_{1}=-16.6, B_{2}=79.5$ from [3]. We consider $u$ as a function of $\xi=x-\beta t$ as in [2].

$$
\beta^{2} \frac{\partial^{2} u}{\partial \xi^{2}}=\frac{\partial}{\partial \xi}\left(B(u) \frac{\partial u}{\partial \xi}\right)-\frac{\partial^{4} u}{\partial \xi^{4}}
$$

Equation (4) is known to have exponentially localized solitonic solutions which propagate without distortion for a finite range of sub-sonic velocities [3].

\subsection{Analytical Solution}

Localized solitonic solutions of (5) are given by (as in [3])

$$
u(\xi)=\frac{2 a_{+} a_{-}}{\left(a_{+}+a_{-}\right)+\left(a_{+}-a_{-}\right) \cosh \left(\xi \sqrt{\left.1-\beta^{2}\right)}\right.}
$$

where $u=a_{ \pm}$are the real roots of the right hand side of the integrated equation, for the velocity range $\beta_{0}<|\beta|<1$ (Fig. 1).

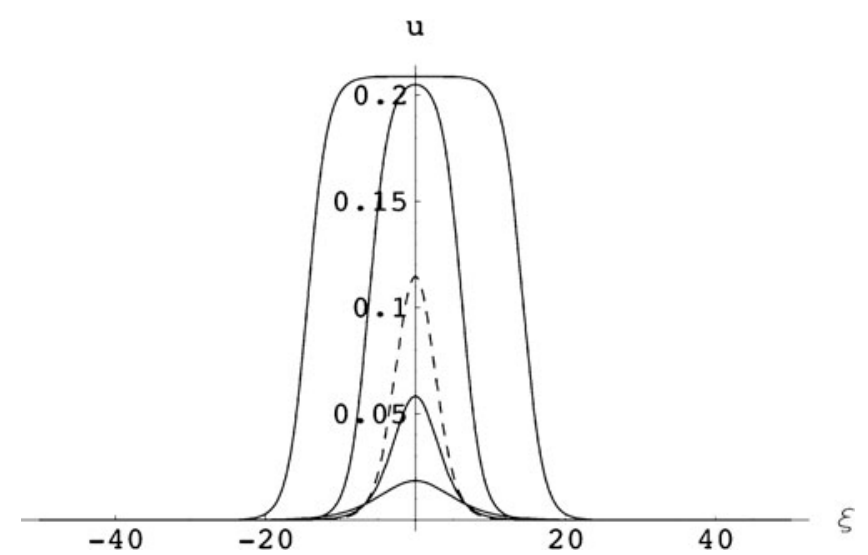

Fig. 1 Soliton profiles for velocities for $\beta=0.95,0.85,0.734671,0.65$ and $\beta_{0}+4 \times 10^{-9}$ respectively from bottom to up. Adapted from [3] 


$$
a_{ \pm}=\frac{-B_{1}}{B_{2}}\left(1 \pm \sqrt{\frac{\beta^{2}-\beta_{0}^{2}}{1-\beta_{0}^{2}}}\right)
$$

- The amplitude of the soliton decreases with the velocity $\beta$.

- The width of the soliton diverges for $\beta \rightarrow \beta_{0}$ and $\beta \rightarrow 1$.

- The soliton has a minimum width at $\beta=0.734671$, shown in dashed line.

\subsection{Numerical Analysis}

To investigate the questions concerning the stability of the solitons of (6), B. Lautrup et.al have considered the model numerically in [3]. In this contribution, the stability of the solitonic solutions for infinitesimal perturbations was carried out along with the effect of dissipation on the soliton propagation. The model, as a system of two first order partial differential equations as mentioned in the reference [3] is used for our numerical consideration (see (8)).

$$
\frac{\partial u}{\partial t}=\frac{\partial v}{\partial x} \quad \frac{\partial v}{\partial t}=\frac{\partial f}{\partial x}
$$

with

$$
f=u+\frac{1}{2} B_{1} u+\frac{1}{3} B_{2} u^{2}-\frac{\partial w}{\partial x} \text { and } w=\frac{\partial u}{\partial x}
$$

To realize the soliton propagation, the model in the above form was solved numerically with Finite Element Method (FEM) in COMSOL Multiphysics 3.5a ${ }^{\circledR}$. The general form in the classical partial differential equation (PDE) mode of COMSOL Multiphysics ${ }^{\circledR}$ was employed with periodic boundary conditions. The analytical solution of $\beta=0.734671$, "minimum width" was chosen as initial condition. Dispersion was found in the solution. The energy of the soliton was found to decrease during the propagation. The algorithm in COMSOL does not yield full numerical stability (Fig. 2).

The stable numerical solution of (7) can be obtained by using a variant of the two-step Lax-Wendroff method as described in [3]. This was executed in C++ [4] and Mathematica ${ }^{\circledR}$ by the authors of [3] and the same is executed here for collision studies.

\section{Collision Studies}

We have investigated the head-on collision of two pulses with identical amplitudes and opposite velocities. It is known that pulses are blocked upon collision [5]. The FitzHugh-Nagumo model [6,7], which is a simplified form of the Hodgkin-Huxley model [8], allows for both the cancellation and penetration of pulses depending 
Fig. 2 Propagation of minimal width soliton. PDE solved with time stepping $\Delta t=0.001$ and $\Delta x=0.1$. The shape of the pulse is not conserved by the numerical algorithm in FEM based Comsol Multiphysics $3.5 \mathrm{a}^{\circledR}$. Length of the periodic lattice has been increased here to depict the change of soliton shape during the propagation

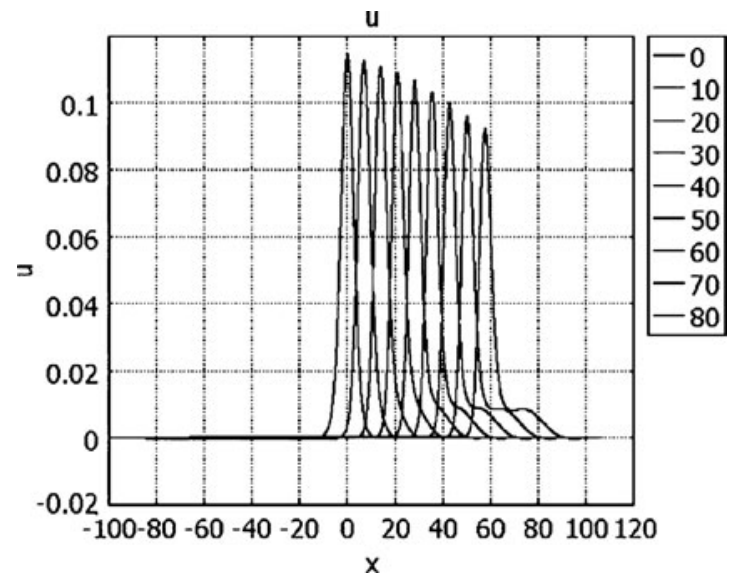

Fig. 3 Collision of two solitons before (a) and after collision (b) shown for $\beta=0.8$. Small amplitude noise travelling ahead of the post-collision pulses for $\beta=0.8$ that carries a very small fraction of the overall energy is obtained. The same was achieved for solitons of different velocity and amplitude

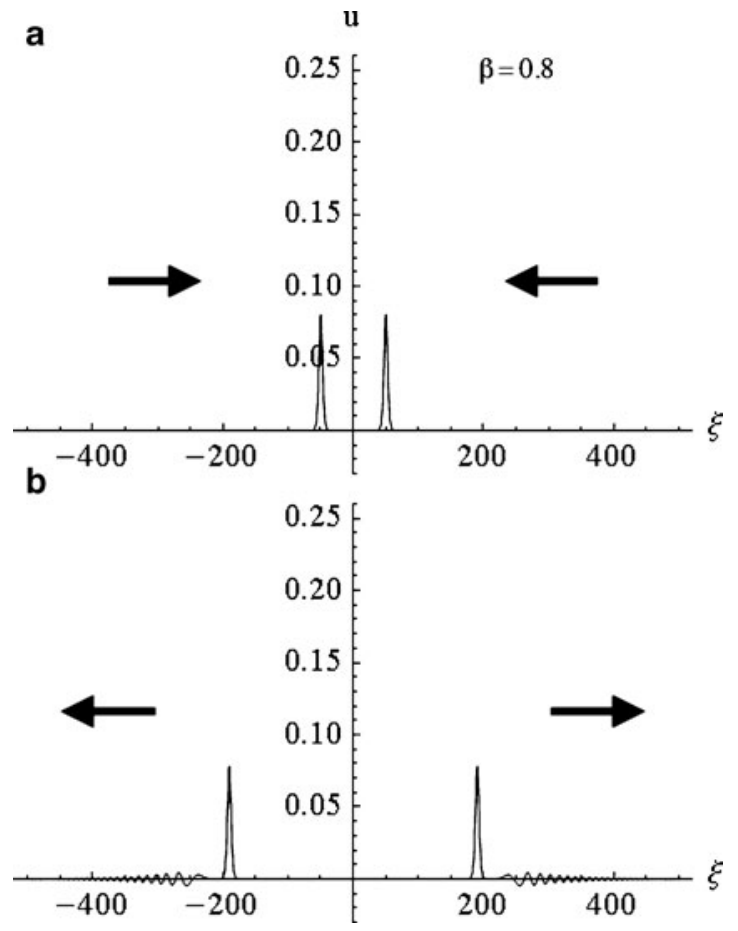

on parameters [9]. Since the soliton model is based on adiabatic and reversible physics without dissipation [10], here we have investigated collisions in the absence of friction. Figure 3 shows two identical solitons with $\beta=0.8$ before and after collision. Small amplitude noise travelled ahead of the post-collision pulses with a very small energy in the order of $\ll 1 \%$ compared to that of the solitons. The same was found for solitary pulses with different velocities and amplitudes. 
The functional dependency of the sound velocity on density was given by (4). It represents a quadratic approximation to the experimental data and yields a satisfactory description in the density regime between solid and liquid membrane state [9]. However, when large amplitude pulses collide, (4) allows the density transiently to exceed the density of the solid phase $(u \approx 0.25)$. Considering this as unphysical, a "soft barrier" at the density of the solid phase is introduced in (4):

$$
B(u)=\left(1+B_{1}(u)+B_{2}\left(u^{2}\right)\right)\left(1+e^{100(x-0.26)}\right)
$$

This modification of (4) is only relevant at the moment for the collision of two large amplitude solitons. The result of such a collision of two solitons with $\beta$ close to the minimum velocity $\beta_{0}$, given by (7), is shown in Fig.4. The soliton fell apart to a sequence of solitons and some additional low amplitude noise. This effect pronounces with the velocity closer to minimum velocity. Such decomposition into several pulses was not seen in the absence of the soft barrier. We compared the largest pulse energy after the collision to the energy before collision (Fig. 5).

The energy density of a soliton has both potential and kinetic energy contributions and can be calculated by using a Lagrangian formalism. (Adapted from [10])

$$
e=\frac{c_{0}^{2}}{\rho_{0}{ }^{A}}\left(\triangle \rho^{A}\right)^{2}+\frac{p}{3 \rho_{0}{ }^{A}}\left(\triangle \rho^{A}\right)^{3}+\frac{q}{6 \rho_{0}{ }^{A}}\left(\triangle \rho^{A}\right)^{4}
$$

Fig. 4 Collision of two solitons before (a) and after collision (b) for $\beta=0.649850822$ (close to maximum amplitude) and the additional condition of a maximum density change of $u=0.25$. The pulse falls apart into several solitary pulses with different amplitude and velocity, and some small amplitude noise
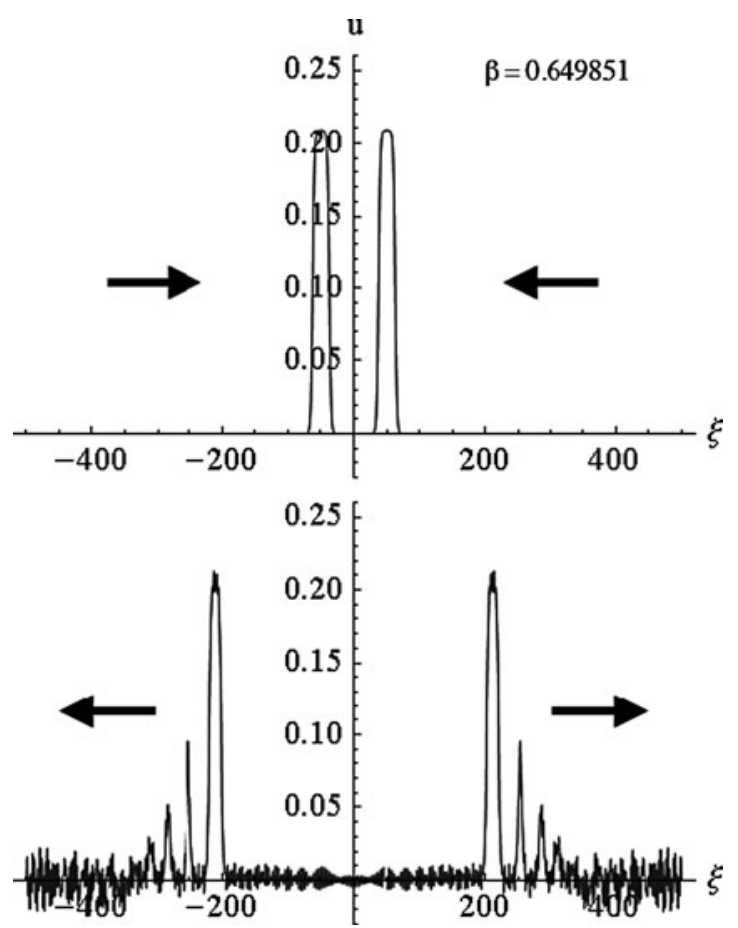


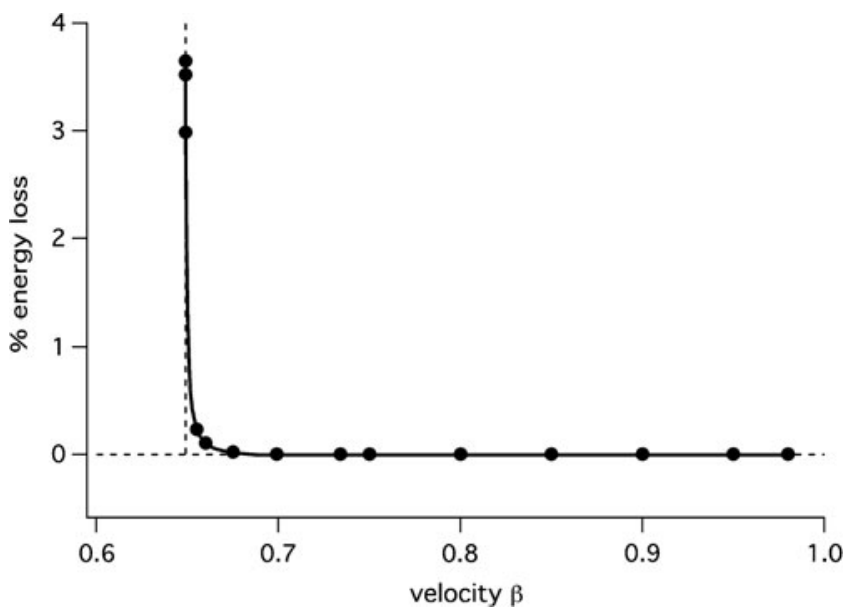

Fig. 5 Energy loss of soliton after collision in \%. The energy content of the largest pulse after collision with the pulse before the collision are compared. Only when the pulses reach their maximum amplitude and minimum velocity, dissipation becomes significant

Even for the near-limiting case the fraction of energy lost into smaller amplitude solitons and small amplitude noise is $<4 \%$ for the most extreme case studied. Thus, we observed most of the energy of the major soliton was conserved in collisions even after a maximum density was enforced.

\section{Conclusion}

The soliton model of nerve pulse propagation with the modified Good-Boussinesq equation [11] is explained. The analytic form of the solitons is given in Sect.2.1. We moved on to numerical analysis of the model Sect. 2.2 to realize the solitary propagation (with periodic boundary conditions) using FEM based software Comsol Multiphysics ${ }^{\circledR}$. Unexpectedly, the numerical solution of the PDE was discrepant from the analytical solution given in [3]. The pulse amplitude was found to be decreasing during the propagation. This can be attributed to an inherent problem of numerical dispersion in the software of Comsol Multiphysics ${ }^{\circledR}$. Simulations were then carried out in $\mathrm{C}++$ and Mathematica ${ }^{\circledR}$ to self-implement the numerical method and to obtain energy-loss less soliton propagation. Finally, the stability of the model is then tested with the aid of collision studies. In the context of our model, pulses pass through each other "almost undisturbed" with the generation of only small amounts of small amplitude noise. If a maximum density is introduced, as seems reasonable for the crystalline lipid matrix, large amplitude solitons can decay into a series of solitons. However, even under these extreme conditions, the bulk of the 
energy remains in the maximum amplitude soliton. Our model does not offer a description of the cancellation of pulses as suggested in other models but opens up a new possibility of passing through almost undisturbed and conserving the maximum energy even upon maximum density enforcement.

Acknowledgements We thank DFG for funding our project "welisa" under GRK 1505/1. Thanks to Andrew. D. Jackson for the meaningful discussions and to Kiran Kumar Sriperumbudur for his thoughtful suggestions.

\section{References}

1. Andersen, S., Jackson, A.D., Heimburg, T.: Towards a thermodynamic theory of nerve pulse propagation. Progr. Neurobiol. 88, 104-113 (2009)

2. Heimburg, T.: Physical properties of biological membranes. In: Bohr, H. (ed.) Encyclopedia of Applied Biophysics, pp. 593-616. Wiley-VCH, Weinheim (2009)

3. Lautrup, B.,Jackson, A.D., Heimburg, T.: The stability of solitons in biomembranes and nerves. physics.bio-ph, arXiv: physics/0510106v1, 2008.

4. Press, W.H., Teukolsky, S.A., Vetterling, W.T., Flannery, B.P.: Numerical recipes in C, 2nd edn. Cambridge University Press, U.K. (1994)

5. Tasaki, I.: Collision of two nerve impulses in the nerve fiber. Biochem. Biophys. Acta. 3, 494497 (1949)

6. FitzHugh, R.: Impulses and physiological states in theoretical models of nerve membrane. Biophys. J. 1, 445-466 (1961)

7. Nagumo, J., Arimoto, S., Yoshizawa, S.: An active pulse transmission line simulating nerve axon. Proc IRE. 50, 2061-2070 (1962)

8. Hodgkin, A.L., Huxley, A.F.: A quantitative description of membrane current and its application to conduction and excitation in nerve. J. Physiol. 117(4), 500-544 (1952)

9. Argentina, M., Coullet, P., Krinsky, V.: Head-on collision of waves in an excitable FitzhughNagumo system:a transition from wave annihilation to classical wave behavior. J. Theor. Biol. 205, 47-52 (2000)

10. Heimburg, T., Jackson, A.D.: On soliton propagation in biomembranes and nerves. Proc. Natl. Acad. Sci. USA 102, 9790-9795 (2005)

11. Manoranjan, V.S., Ortega, T., Sanz-Serna, J.M.: Soliton and antisoliton interactions in the good Boussinesq equation. J. Math. Phys. 29(9), 1964-1968 (1988) 